\title{
Type I Interferon Regulates Respiratory Virus Infected Dendritic Cell Maturation and Cytokine Production
}

\author{
BRIAN D. RUDD, ${ }^{1}$ GARY D. LUKER, ${ }^{2}$ KATHRYN E. LUKER, ${ }^{2}$ RAY S. PEEBLES, ${ }^{3}$ \\ and NICHOLAS W. LUKACS ${ }^{1}$
}

\begin{abstract}
Activation of dendritic cells (DCs) by viruses is critical for both innate and adaptive immune responses. In this report, we investigated the role of type I interferon (IFN) in the activation of DCs by respiratory syncytial virus (RSV). Using DCs from type I IFNR ${ }^{-/-}$mice, these studies indicate that maturation, including upregulation of co-stimulatory molecules and optimal cytokine production, by RSV infection was dependent on type I IFN receptor signaling. Subsequently, studies using DCs from wild type mice demonstrate that continued production of type I IFN during later stages of DC maturation could alter their activation profiles. IFN- $\alpha$ and IFN- $\beta$ were upregulated in DCs grown from bone marrow of wild type mice after infection with RSV. In order to determine their function in competent DCs, blocking antibodies were used to specifically inhibit IFN- $\alpha / \beta$. The data demonstrate that production of IFN- $\beta$, but not IFN- $\alpha$, in RSV-infected wild type DCs promotes chemokine production and toll-like receptor (TLR) expression, while limiting IL-12 production. The inhibition of IL-12p70 by IFN- $\beta$ correlated with suppressed IL-12p40 expression levels. Furthermore, the addition of recombinant IFN- $\beta$ potently inhibited IL-12p40 expression in mature DC subsets during RSV infection, while only the highest dose of IFN- $\alpha$ had any inhibitory effect. Together, our studies provide insight into the complex regulation of DC maturation and IL-12 production coordinated by type I interferons in RSV-infected dendritic cells, and demonstrate that type I IFN has specific roles depending upon the stage of DC maturation.
\end{abstract}

\section{INTRODUCTION}

$\mathbf{R}$ ESPIRATORY SYNCYTIAL VIRUS (RSV) is the leading cause of lower respiratory tract infections in infants. Severe RSV infections early in life have been linked to the later development of pulmonary problems, even years after the infection has been resolved (1). The development of an appropriate immune response during viral infections relies on early events in innate immune cells to shape the immediate environment and dictate the course of infection, but may also alter the lung environment and its reactions to subsequent immune responses.

Dendritic cells (DCs) are antigen-presenting cells located at the portals of pathogen entry that link innate and adaptive immunity. Dendritic cells and phagocytes rapidly produce type I interferons and IL-12 in response to viral infections. However, the interaction of type I interferons and IL-12 in virally infected dendritic cells is still poorly understood. Type I interferons consist of a single IFN- $\beta$ gene and a large family of IFN- $\alpha$ genes.

\footnotetext{
${ }^{1}$ Department of Pathology, ${ }^{2}$ Department of Radiology, University of Michigan Medical School, Ann Arbor, Michigan, and ${ }^{3}$ Vanderbilt University School of Medicine, Department of Medicine, Vanderbilt University, Nashville, Tennessee.
} 
Historically, interferons were known to render cells resistant to some viruses and were considered part of the first wave of host defense (2). While these cytokines may play a critical role in antiviral defense, many immunoregulatory functions in both innate and adaptive immunity have been described. It has recently been shown that type I interferons may also promote the induction of interferon-stimulated genes (ISGs), natural killer (NK) cell cytotoxicity, T-cell polarization, and DC maturation (3). In this study, we aimed to define the role of type I interferons in regulating DC activation by RSV.

In response to viruses, a subset of type I interferons are rapidly secreted and bind the type I interferon receptor in autocrine and paracrine fashion (4). Upon binding to its receptor, the JAK-STAT pathway is engaged, leading to the subsequent upregulation of all type I interferons and ISGs. This positive feedback loop has also been implicated in the cross-regulation of IL-12 in activated dendritic cells (5).

In this study, we first aimed to describe the functional role of type I interferon signaling in the activation of DCs by RSV, and demonstrate that the type I interferon signaling axis is required for upregulation of co-stimulatory molecules and production of cytokines and chemokines. These studies subsequently defined the relative contribution of IFN- $\beta$ in RSV-infected DCs. The release of IFN- $\beta$ by RSV-infected DCs was not essential for upregulation of co-stimulatory molecules, but it played a critical role in regulating levels of cytokines and chemokines. Interestingly, our data demonstrate that IFN$\beta$ had a positive impact on CXCL10 and CCL2 levels, but negatively affects IL-12 production. By specifically targeting IFN- $\beta$, our results define an opposing role for IFN- $\beta$ in RSV-infected dendritic cells, as well as demonstrate that type I IFNs can have divergent effects on DC activation depending upon the maturation state of the DC. These data suggest that although activation via the type I IFN receptor is crucial for maturation of the dendritic cell, IFN- $\beta$, but not IFN- $\alpha$, plays the primary role in regulation of bone marrow-derived dendritic cell (BMDC) cytokine production.

\section{MATERIALS AND METHODS}

\section{Reagents, virus, and infections}

Neutralizing antibodies against IFN- $\alpha$ (RMMA-1), IFN- $\beta$ (RMMB-1), and control were purchased from PBL Biomedical Laboratories (Piscataway, NJ). Recombinant murine IFN- $\alpha$ and IFN- $\beta$ and the ELISA kits were also purchased from PBL Biomedical Laboratories. The A2 strain of RSV was propagated in Hep2 cells. The viral titer for virus was determined by RSV-specific antibod- ies to visualize the plaques in infected cultures and enumerate positive plaques in serially diluted Vero cell cultures. The titer for each virus was determined within a month of performing the studies and the virus stocks were stored at $-80^{\circ} \mathrm{C}$ until use. All stocks were shown to be free of mycoplasma contamination using a detection kit from Cambrex (East Rutherford, NJ). UV inactivation of virus was performed in a laminar flow hood for $30 \mathrm{~min}$ with the virus being $\sim 5$ inches from the UV light source. The DCs were incubated with RSV grown and titered within the previous month and applied to the cultures at a multiplicity of infection of $1.0(\mathrm{MOI}=1.0)$.

\section{Generation of bone marrow-derived dendritic cells}

Mice deficient in type I IFN receptor (IFN-I $\mathrm{R}^{-/-}$) expression were maintained and provided by Gary and Kathryn Luker (University of Michigan, Ann Arbor, MI). 129/SvEv mice (wild type) were purchased from Taconic (Hudson, NY). Anti-type IFN experiments were initially performed with BMDCs generated from C57BL/6 mice (Jackson Laboratories, Bar Harbor, ME), but they were repeated in 129/SvEv DCs with comparable results. Bone marrow was harvested from mice and seeded in tissue culture flasks in RPMI 1640-based complete media with $20 \mathrm{ng}$ granulocyte-macrophage colony-stimulating factor (GM-CSF)/mL (R\&D Systems, Minneapolis, MN). The cells were fed after $3 \mathrm{~d}$ and loosely adherent cells were collected after $6 \mathrm{~d}$ and incubated with anti-CD11c antibody coupled to magnetic beads (Miltenyi Biotec, Auburn, CA). The BMDCs were purified using positive selection for CD11c+ cells by running the cell suspension through a magnetic column. The next day, CD11c+ BMDCs were infected with RSV (MOI $=1.0$ ) for $2 \mathrm{~h}$, washed, and resuspended in fresh media for the indicated times. The cells were then assessed for gene and protein expression.

\section{Quantification of cytokine expression}

Protein levels of IL-12p70 were quantitated using a Bio-Plex bead-based cytokine assay purchased from BioRad Laboratories (Hercules, CA). RNA was isolated using Trizol. Levels of IL-12p40 and CCL2 were assessed using qPCR analysis (TaqMan; Applied Biosystems, Foster City, CA) with predeveloped primers and probe sets from PE Biosystems (Foster City, CA). Primers for CXCL10 and RSV G protein were custom designed as previously described (6). Primer sequences for IFN- $\alpha$ and IFN- $\beta$ have been previously described (7). qPCR analysis of IFN was performed using SYBR green I dye. Results were normalized to glyceraldehyde-3-phosphate dehydrogenase (GAPDH) expression and presented as fold increase in mRNA expression compared to the level de- 
tected at day 0 using the comparative $\mathrm{C}_{\mathrm{T}}$ method $\left(\Delta \Delta \mathrm{C}_{\mathrm{T}}\right)$ established by Applied Biosystems. As outlined by the literature, this technique provides a standardized methodology that is quantitative based upon a standard housekeeping gene expression for sample comparisons.

\section{Flow cytometric analysis}

Cells were stained with the indicated antibodies (BD Pharmingen, San Diego, CA) that were specific for costimulatory molecules and analyzed using a fluorescenceactivated cell sorter (FACS) Calibur and Cell Quest software (BD Biosciences, San Jose, CA). Isotype control antibodies were used to demonstrate specificity of our staining and to establish the criteria for our flow cytometry populations.

\section{Statistical analysis}

All results are expressed as mean \pm SE. Statistical significance was calculated by ANOVA followed by the student's Neuman Kühl post-test to calculate the $p$ value. Significance was determined at the level of $p<0.05$.

\section{RESULTS}

\section{Type I interferon receptor is essential for} $R S V$-induced dendritic cell maturation

To examine the kinetics of IFN- $\alpha / \beta$ production in RSV-infected dendritic cells from wild type mice, we purified $\mathrm{CD} 11 \mathrm{c}+\mathrm{BMDCs}$ and infected $(\mathrm{MOI}=1.0)$ these cells for 8 and $24 \mathrm{~h}$ post-infection (hrpi). RNA was isolated at the given time points and levels of IFN- $\alpha$ and IFN- $\beta$ were examined by qPCR. Following RSV infection, IFN $-\alpha$ and IFN- $\beta$ were highly induced at 8 hrpi and were lower by $24 \mathrm{hrpi}$ (Fig. 1). UV-inactivated RSV was incapable of driving type I IFN production, indicating live RSV was again required for type I interferon synthesis in RSV-infected dendritic cells (Fig. 1A). Interestingly, IFN- $\beta$ was induced at $\sim 4$-fold higher levels than IFN $-\alpha$, and IFN- $\beta$ expression continued to be detectable at $24 \mathrm{hrpi}$. We also examined the level of type I IFN protein expressed after 24 hrpi from wild type DCs (Fig. 1B). Both IFN- $\alpha$ and IFN- $\beta$ levels were undetectable unless infected with RSV. IFN- $\beta$ was produced at 10 -fold higher levels than IFN- $\alpha$ based on the specific protein levels (Fig. 1B).

Upon maturation, dendritic cells upregulate co-stimulatory molecules and activate $\mathrm{T}$ cells in the adaptive immune response. Since a number of reports have shown a correlation between type I interferon and the maturation of dendritic cells by viruses $(8,9)$, we first asked whether type I interferon was required for upregulation of co-stimulatory molecules in RSV-infected dendritic cells. To this end, we infected bone marrow-derived dendritic cells (BMDCs) from wild type and IFN-I $\mathrm{R}^{-1-}$ mice with RSV. After 24 hours, the DCs were analyzed by flow cytometry for levels of CD40 and CD86 to determine the maturation status. RSV significantly upregulated both CD40 and CD86 expression in wild type DCs (Fig. 2). However, upregulation of co-stimulatory molecules was severely impaired in IFN-I R ${ }^{-/-}$DCs (Fig. 2), demon-
A

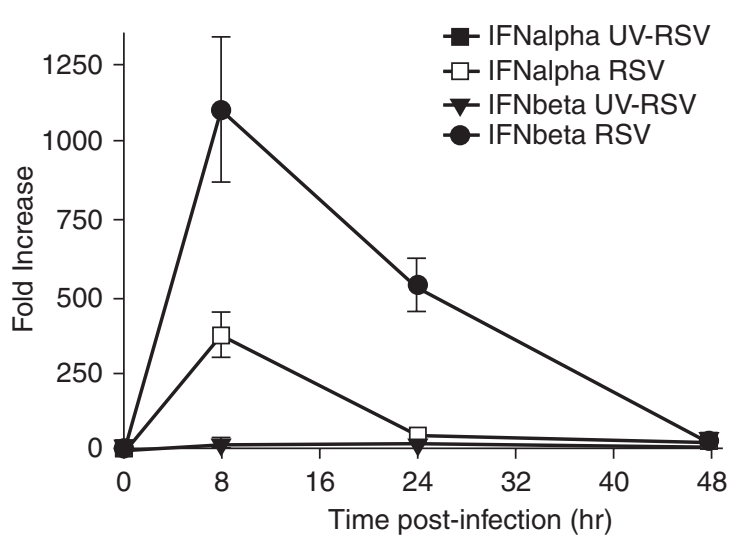

B

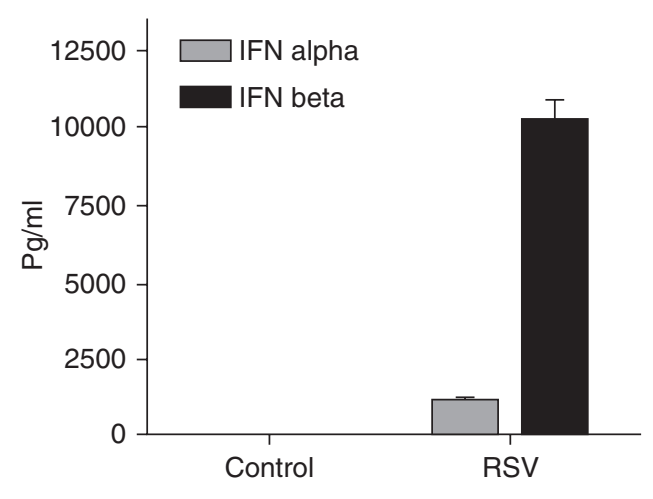

FIG. 1. Type I IFNs are upregulated in DCs infected with RSV. Wild type DCs were infected with live RSV, UV-inactivated RSV, or incubated with media alone for 8, 24, or 48 h. (A) At the indicated time points, expression levels of IFN- $\alpha 4$ or IFN- $\beta$ were measured by qPCR and expressed as the mean fold change relative to media controls. (B) To further assess the relative production of type I IFNs protein levels were assessed in DCs after $24 \mathrm{~h}$ of RSV infection. Data are representative of three repeat experiments, with $\mathrm{n}=3$ per treatment per experiment. 

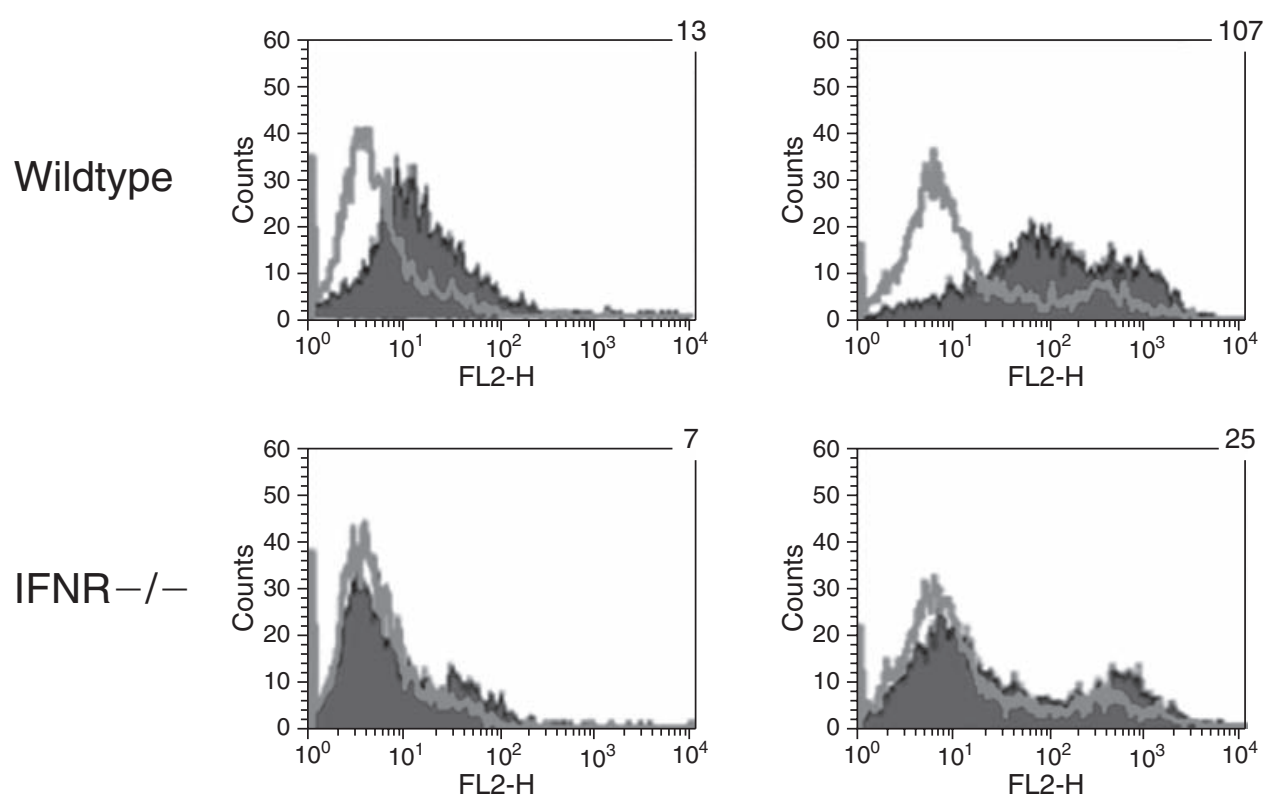

FIG. 2. Type I interferon signaling is required for RSV-induced maturation of DCs. DCs derived from wild type and IFN-I $\mathrm{R}^{-1-}$ were infected with RSV for $24 \mathrm{~h}$. Expression of co-stimulatory molecules CD40 and CD86 were analyzed by FACS. Open curves depict unstimulated DCs; shaded histograms represent RSV-infected DCs. The mean fluorescence intensity (MFI) for each condition is indicated in the upper corner. Data are representative of three repeat experiments with similar results.

strating that type I interferon is essential for virus-induced maturation in RSV-infected dendritic cells. UV-inactivated RSV was incapable of upregulating CD40 and CD86 in wild type DCs, indicating that replication-competent RSV was required to promote DC maturation (data not shown).
Previous reports demonstrated that type I interferon signaling in the early phase of DC maturation was essential to sustain inflammatory cytokine production in the later phases (9). Given the profound defect in CD40 and CD86 expression in IFN-I $\mathrm{R}^{-/-}$DCs, we next examined
A

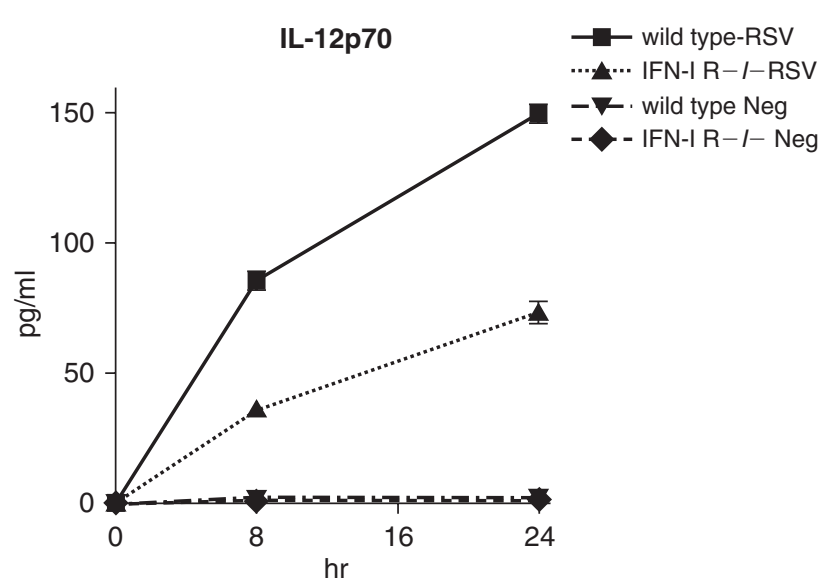

B

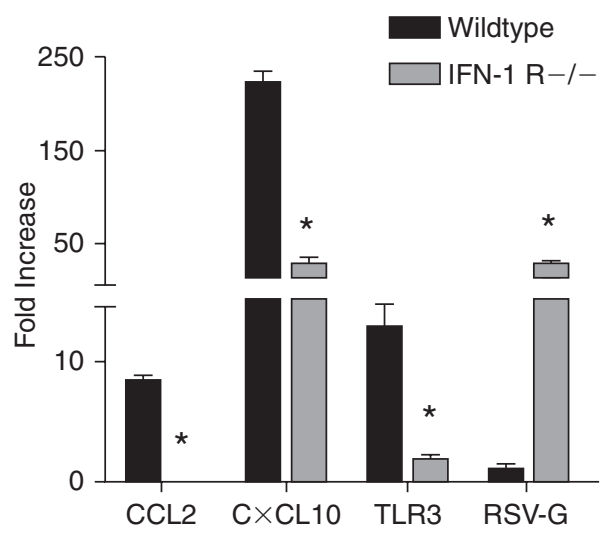

FIG. 3. Type I interferon signaling is required for optimal cytokine/chemokine production in RSV-infected DCs. DCs derived from wild type and IFN-I R $\mathrm{R}^{-1-}$ mice were infected with RSV. (A) At 8 and $24 \mathrm{~h}$, protein levels of IL-12p70 in the supernatant were determined. (B) Expression levels of CXCL10, CCL2, TLR3, and RSV-G were measured using qPCR and the relative fold increase compared to RSV-infected wild-type DCs. Data are representative of three repeat experiments, with $\mathrm{n}=3$ per treatment per experiment. Each time point represents the mean $\pm \mathrm{SE}(* p<0.05)$. 
the role of type I interferon receptor for IL-12 production in RSV-infected BMDCs obtained from wild type and IFN-I $\mathrm{R}^{-1-}$ mice. Interestingly, IFN-I $\mathrm{R}^{-/-} \mathrm{DCs}$ infected with RSV had significantly reduced levels of IL12 p70 protein compared to wild type DCs (Fig. 3A). These results indicate a partial requirement for type I interferon signaling in optimal IL-12 production in RSVinfected DCs.

Interferons have also been shown to induce specific chemokines involved in antiviral immunity (10). Next we compared mRNA levels of CXCL10 and CCL2 in wild type and IFN-I R $\mathrm{R}^{-/-}$DCs. Induction of both CXCL10 and CCL2 were almost entirely abrogated in IFN-I $\mathrm{R}^{-1-}$ DCs infected with RSV (Fig. 3B). In addition, when we examined TLR3 expression of the IFN-I $\mathrm{R}^{-/-}$dendritic cells, they were deficient in their ability to increase TLR3 expression (Fig. 3B). While one possible interpretation of these results might be that the IFN-I R ${ }^{-1-}$ DCs were not infected, our assessment of RSV-G protein expression demonstrated that there was actually an increase in RSV gene expression in the DCs from IFN-I $\mathrm{R}^{-1-}$ mice. These results indicate that the induction of chemokines and important innate sensing molecules such as TLR3 in

A CXCL10

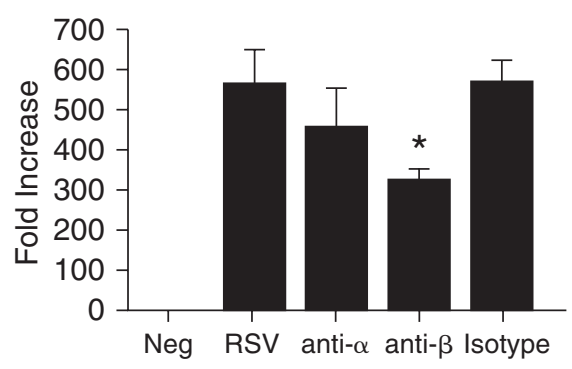

C

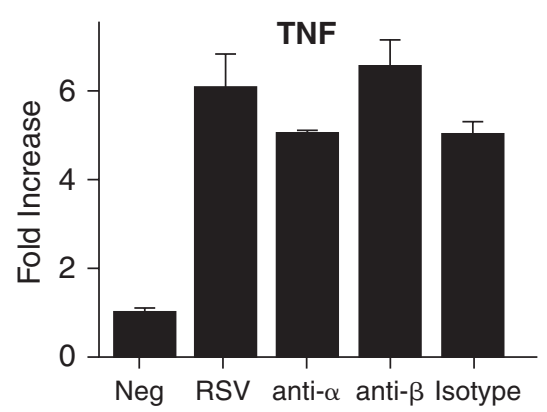

RSV-infected DCs is dependent on activation by the type I interferon receptor.

\section{Type I interferon differentially regulates cytokine production during dendritic cell maturation}

Since type I interferon receptor signaling was essential for the induction of interferon-dependent chemokines, we asked whether RSV-induced chemokine production was affected by the release of IFN- $\alpha$ or IFN- $\beta$ by infected DCs. In particular, CXCL10 and CCL2 have previously been shown to be type I IFN inducible during viral infection as was recently reviewed (11). We compared mRNA levels of CXCL10 and CCL2 from RSVinfected cells $(\mathrm{MOI}=1.0)$ pretreated with a pan-inhibitory anti-IFN- $\alpha$ or a specific anti-IFN- $\beta$. Pretreatment of DCs with anti-IFN suppressed levels of IFN activity $>60 \%$ (data not shown). qPCR analysis of CXCL10 and CCL2 from cells treated with anti-IFN- $\beta$ showed a dramatic reduction in those chemokines compared to untreated and isotype-control treated cells, indicating that IFN- $\beta$ mediates chemokine production in RSV-infected dendritic cells (Fig. 4). Antibodies to IFN-

B

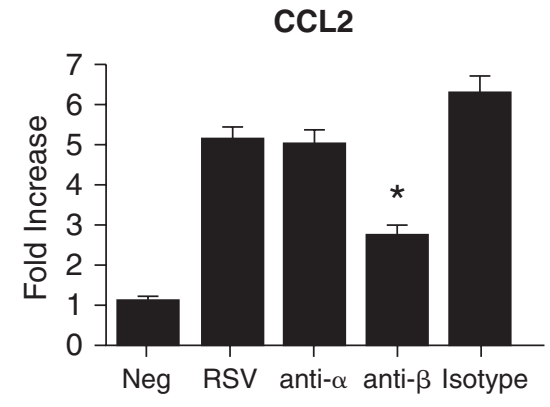

D

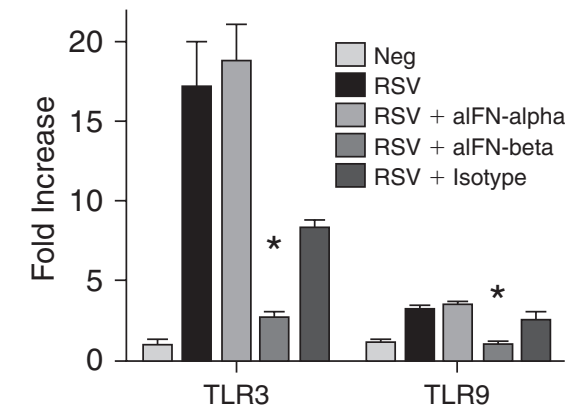

FIG. 4. IFN- $\beta$ is essential for chemokine production in RSV-infected DCs. DCs were pretreated with either anti-IFN- $\alpha$, antiIFN- $\beta$ (20 $\mu \mathrm{g} / \mathrm{mL})$, isotype control $(20 \mu \mathrm{g} / \mathrm{mL})$, or medium prior to RSV infection. The antibodies were left in the media and cells were infected with RSV. At 24 hrpi, RNA levels of CXCL10 (A), CCL2 (B), and TNF (C) were analyzed by qPCR and expressed as the fold change relative to media controls. Similarly, the mRNA was also examined for expression of TLRs (D), which are upregulated after RSV activation. Data are representative of three repeat experiments, with $\mathrm{n}=3$ per treatment per experiment. Each time point represents the mean \pm SE. $(* p<0.05)$ 
$\alpha$ did not reduce any of the same responses observed by blocking IFN- $\beta$. The ability of the antibody against IFN- $\alpha$ to neutralize was verified using recombinant IFN- $\alpha$ on uninfected DCs (data not shown). In contrast, when we examined another often-produced DC-derived cytokine, tumor necrosis factor (TNF), no alteration was detected after blockade of IFN- $\beta$ (Fig. 4C). In addition to cytokine production, the activation and maturation of DCs by type I IFN also can regulate the expression of additional innate molecules such as toll-like receptors (TLRs) that recognize viral nucleic acid and further allow maturation of DCs. In the present studies we examined the expression of TLR3, that recognizes dsRNA, and TLR9, that recognizes unmethylated $\mathrm{CpG}$, and were both upregulated (Fig. 4D). The data illustrate that by neutralizing IFN- $\beta$, but not IFN- $\alpha$, the expression of these TLRs are both significantly reduced after RSV infection.

The data in the IFN-I $\mathrm{R}^{-/-}$DCs described above indicate dependence on type I IFN for IL-12 production. To determine the specific role of type I IFN in IL-12 production, we also measured IL-12 levels in RSV-infected
DCs treated with anti-IFN- $\alpha / \beta$. Only neutralization of IFN- $\beta$ resulted in significant upregulation of IL-12p70 protein, indicating that the release of IFN- $\beta$ in RSV-infected BMDCs profoundly impairs the ability of RSV to synthesize IL-12 (Fig. 5A). The mechanism of IL-12 inhibition by IFN- $\beta$ has been attributed to the transcriptional downregulation of the IL-12p40 chain (12). To test this possibility, we next measured the upregulation of IL12p40 mRNA in RSV-infected DCs treated with antitype I IFN antibodies. The accumulation of IL-12p40 mRNA in anti-IFN- $\beta$-treated DCs correlated with the elevated levels of IL-12p70 protein (Fig. 5A and 5B). These findings were also verified when we infected DCs in the presence of increasing amounts of IFN- $\alpha$ or IFN- $\beta$. When the recombinant cytokines were used, although IFN- $\alpha$ was able to reduce IL-12p40 expression at the highest dose, it was not as effective as IFN- $\beta$, which induced a dose-dependent reduction in IL-12p40 mRNA (Fig. 5C and 5D). When IL-12p35 subunit mRNA was examined in these same studies, no alteration after any of the treatments compared to control treated cells was observed (data not shown).
A

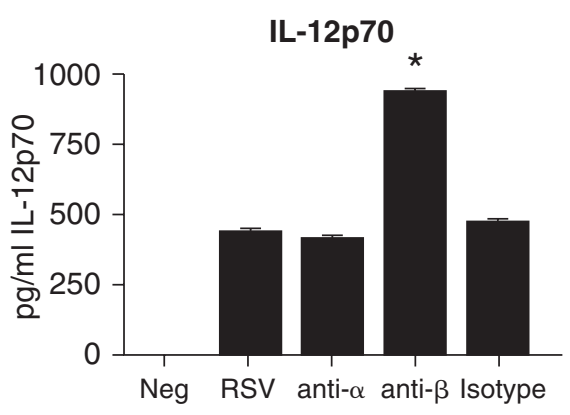

C

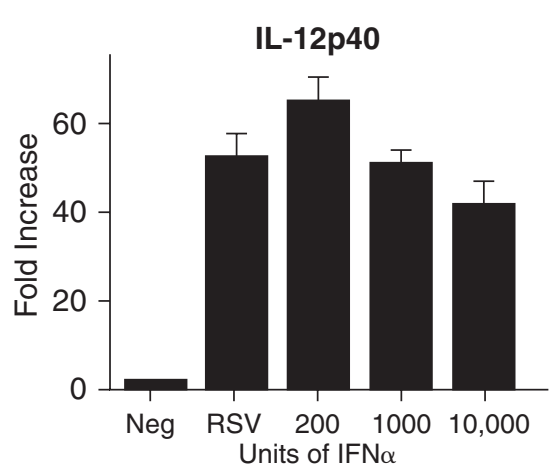

B

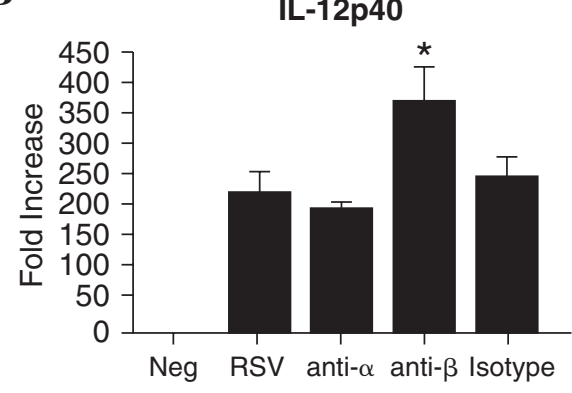

D IL-12p40

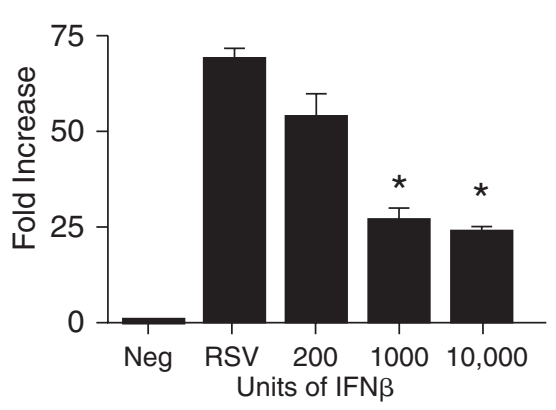

FIG. 5. Neutralization of IFN- $\beta$ upregulates IL-12 in DCs infected with RSV. DCs were pretreated with anti-IFN- $\beta$ (20 $\mu \mathrm{g} / \mathrm{mL}$ ), isotype control (20 $\mu \mathrm{g} / \mathrm{mL})$, or medium, and infected with RSV for $24 \mathrm{~h}$. Protein levels of IL-12p70 (A) and RNA levels of IL-12p40 (B) were determined as previously described. Increasing concentrations of recombinant murine IFN- $\alpha$ (C) or IFN- $\beta$ (D) were also added to DCs with RSV, and expression levels of IL-12p40 were determined at 24 h. Data are representative of three repeat experiments, with $n=3$ per treatment per experiment. Each time point represents the mean $\pm \mathrm{SE}(* p<0.05)$. 


\section{Blocking IFN- $\beta$ does not alter co-stimulatory molecules on RSV-infected dendritic cells}

Our data with IFN-I $\mathrm{R}^{-1-}$ mice also suggest that DC maturation does not occur by direct signaling by RSV infection, but requires the autocrine/paracrine type I interferon-signaling pathway. To examine the relative contribution of IFN- $\beta$, we next evaluated the ability of RSV-infected DCs pretreated with anti-IFN- $\beta$ to upregulate CD40 and CD86. However, neutralization of IFN- $\beta$ did not significantly impair DC expression of these two co-stimulatory molecules by itself, since they were similar to control DCs infected with RSV (Fig. 6). Together, neutralization of IFN- $\beta$ had no discernable affect on DC co-stimulation in RSV-infected DCs, but it is necessary for optimal production of specific cytokines, chemokines, and TLRs.

\section{DISCUSSION}

The above-described experiments establish that type I interferon receptor signaling is essential for the upregulation of co-stimulatory molecules and optimal cytokine/chemokine production in RSV-infected DCs early in the maturation process. When type I interferon production was assessed it was observed that a significant upregulation of both IFN- $\alpha$ and IFN- $\beta$ was induced by RSV, with IFN- $\beta$ expressed at eight- to 10 -fold higher levels when we assessed the protein. The inability of RSV
CD40

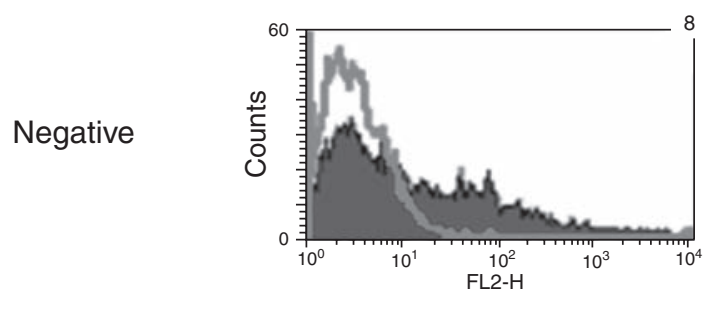

RSV

RSV anti-IFN $\beta$

RSV Isotype
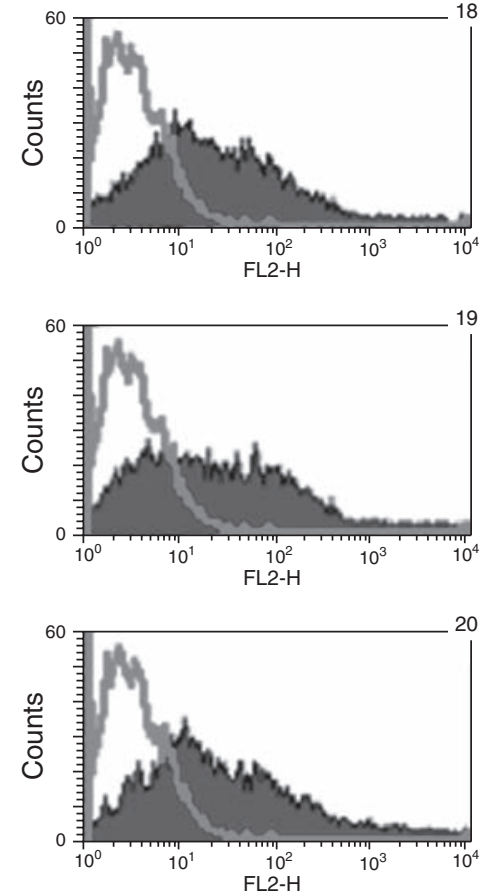

CD86
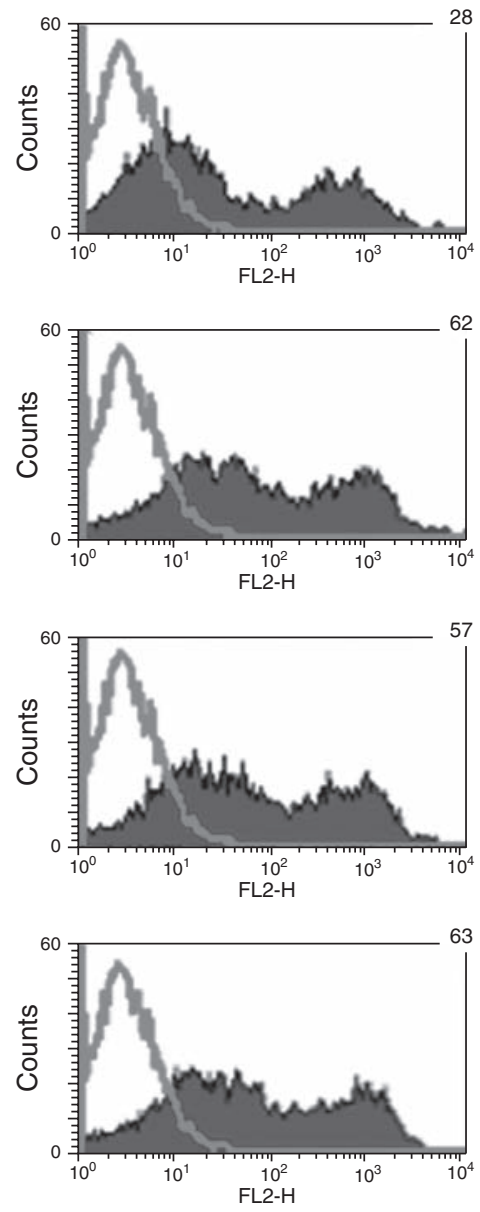

FIG. 6. IFN- $\beta$ is not required for RSV-induced maturation of wild type DCs. DCs were pretreated with anti-IFN- $\beta$ (20 $\mu \mathrm{g} / \mathrm{mL}$ ), isotype control (20 $\mu \mathrm{g} / \mathrm{mL})$, or medium, and infected with RSV for $24 \mathrm{~h}$. Expression levels of the co-stimulatory molecules CD40 and CD86 were analyzed by FACS. Open curves depict isotype controls; shaded histograms represent each treatment group. The MFI for each condition is indicated in the upper corner. Data are representative of three repeat experiments with similar results. 
to upregulate CD40 and CD86, chemokines, and optimize IL-12p70 levels, in DCs from the type I IFN receptor knockout mice we demonstrated the importance of this signaling molecule during the initial stages of RSV infection. Given the global impairments in the activation of IFN-I R ${ }^{-1-}$ DCs infected with RSV, the function of type I IFNs was further assessed in DCs from wild type mice using neutralizing antibodies specific for IFN- $\alpha$ or IFN- $\beta$. Similar to RSV-infected IFN-I $\mathrm{R}^{-1-}$ DCs, chemokine production was significantly downregulated in anti-IFN- $\beta$-treated cells. The most interesting aspect of these studies was that blocking IFN- $\beta$ has no effect on the expression of co-stimulatory molecules, but it augments IL-12p70 production. Thus, while the expression of co-stimulatory molecules and IL-12p70 production is severely impaired in IFN-I R $\mathrm{R}^{-/-}$DCs, DCs treated with anti-IFN- $\beta$ expressed similar levels of co-stimulatory molecules, but enhanced IL-12p70 production. These observations can be explained by at least two possible mechanisms: First, type I interferon signaling is important in maturing RSV-infected DCs to the point of being able to produce IL-12, but continued production of IFN- $\beta$ regulates IL-12 in maturing DCs. Alternatively, RSV might require type I interferon signaling to initiate the induction of IL-12p35 (5), but later regulate IL-12p40 production in activated DCs via a negative feedback loop involving type I IFN $(12,13)$. Thus, these data demonstrate that DCs have the ability to modify their own responses by continued production of IFN- $\beta$ during later stages of DC maturation.

Our recognition that IL-12 regulation is linked to DC maturation is based on the observation that optimal IL12 levels are only produced in DCs that are capable of undergoing maturation. While the type I interferon receptor is required for optimal IL-12p70 production in DCs stimulated with a combination of TLR ligands that are able to induce IL-12p70 (5), IFN-I R ${ }^{-1-}$ DCs stimulated with dsRNA or lipopolysaccharide do not mature (14). When IFN- $\beta$ was specifically targeted in RSV-infected DCs, the ability to upregulate co-stimulatory molecules is not impaired. The key finding is that IFN- $\beta$ being produced in DCs that are capable of undergoing maturation is an important regulator of cytokine production by both limiting IL-12p70 and by augmenting chemokine production. While the implications of these observations are not completely clear, they may have significant impact on T-lymphocyte recruitment and differentiation during antigen presentation. Of considerable interest is the fact that when IFN- $\alpha$ was blocked a similar effect was not observed. This may be due to the fact that the majority of type I IFN expressed in these BMDC studies was IFN- $\beta$. However, rIFN- $\alpha$ was not able to regulate IL-12p40 expression in a similar manner as IFN- $\beta$ upon RSV infection. This result might explain how pDCs, which are the primary producers of IFN- $\alpha$, may participate in the maturation of $\mathrm{mDCs}$ within the lymph nodes without altering IL-12 production, and therefore aid in the overall antiviral response (15).

Numerous signaling mediators in the type I interferon loop that regulate IL-12p70 production have been described and include STAT1 and IRF3 $(5,16)$. The decreases in IL-12p70 in IFN-I R ${ }^{-1-}$, STAT1 ${ }^{-1-}$, and $\mathrm{IRF}^{-1-}$ DCs was largely attributed to impaired IL12 p35 production. Recent evidence suggests that IRF3 is a key transcription factor in IL-12p35 production (16). While induction of IRF3 occurs through direct TLR signaling pathways, it may be further amplified by signaling through the type I interferon receptor. Thus, lower levels of IL-12p70 in RSV-infected IFN-I R ${ }^{-1-}$ DCs may represent only the direct IRF3 pathway via TLR signaling, and be missing the amplified signal that occurs in wild type DCs through the type I interferon receptor. However, high levels of type I inteferons have also been demonstrated to inhibit IL-12p70 by limiting IL-12p40 production in both human and murine dendritic cells $(12,13,17,18)$. While IL-12p40 is inducible, it is commonly produced in large excess. This may explain why high levels of interferons are required to downregulate IL-12p40 to quantities that would begin to limit IL-12p70 levels. While we see little or no induced p19 mRNA levels or IL-23 production after RSV infection (data not shown), the regulation of IL-12p40 by type I IFN during other responses could also affect IL-23 production as well. Interestingly, while the anti-IFN- $\beta$ treatment allowed enhanced production of IL-12p70, there was a reduction in TLR3 and TLR7 expression by the RSV-infected DCs. The implications of this opposing regulation of TLRs and IL-12 are also unclear, but may relate to the overall maturation state of the DC as it becomes less responsive to external stimuli by downregulating TLRs at the same time that is producing more IL-12 to skew the ensuing virus-specific adaptive responses. Our data suggest that sufficient amounts of IFN- $\beta$ are produced in RSV-infected DCs to limit IL-12p70 production. The inhibition of IL-12p70 by IFN- $\beta$ correlated with suppression of only IL-12p40, since IL-12p35 levels were similar among all treatment groups. These latter studies are consistent with previous observations that demonstrated that endogenous type I IFN produced in LCMV- and MCMV-infected mice profoundly inhibited IL-12 produced by DCs $(19,20)$. Consistent with the data that show the ability of IFN- $\alpha$ to regulate IL- 12 were studies examining production of IFN- $\alpha$ by pDCs in MCMV-infected mice, that showed inhibited IL-12 levels in nonpDC subsets $(19,21)$.

Studies indicate that effective viral responses correlate with sufficient IL-12 production by immune cells $(22,23)$. In studies more specific to RSV infection, investigators 
used IL-12p $40^{-1-}$ mice, and demonstrated a role of IL$12 \mathrm{p} 40$ in limiting viral replication, inflammation, mucus production, and Th2 cytokine production $(24,25)$. Previous results indicated that treatment of RSV-infected mice with anti-IL-12 resulted in increased AHR and mucus expression mediated by IL-13 (26,27). These studies further support seminal studies linking type I IFNs with immune responses in RSV infection (28). The current study suggests that excessive IFN- $\beta$ might contribute to pulmonary pathology by altering IL-12 levels during severe $\mathrm{RSV}$ responses, and limit development of effective antiviral responses. This latter suggestion may be intriguing and testable during a normal RSV infection in wild type mice.

\section{REFERENCES}

1. Sigurs N, Gustafsson PM, Bjarnason R, Lundberg F, Schmidt S, Sigurbergsson F, and Kjellman B: Severe respiratory syncytial virus bronchiolitis in infancy and asthma and allergy at age 13. Am J Respir Crit Care Med 2005; 171:137.

2. Isaacs A, and Lindenmann J: Virus interference. I. The interferon. Proc R Soc Lond B Biol Sci 1957;147:258.

3. Biron C: Interferons alpha and beta as immune regulatorsA new look. Immunity 2001;14:661.

4. Levy DE, Marie I, Smith E, and Prakash A: Enhancement and diversification of IFN induction by IRF-7-mediated positive feedback. J Interferon Cytokine Res 2002;22:87.

5. Gautier G, Humbert M, Deauvieau F, et al.: A type I interferon autocrine-paracrine loop is involved in toll-like receptor-induced interleukin-12p70 secretion by dendritic cells. J Exp Med 2005;201:1435.

6. Miller AL, Bowlin TL, and Lukacs NW: Respiratory syncytial virus-induced chemokine production: linking viral replication to chemokine production in vitro and in vivo. Infect Dis 2004;189:1419.

7. Karaghiosoff M, Steinborn R, Kovarik P, et al.: Central role for type I interferons and Tyk2 in lipopolysaccharideinduced endotoxin shock. Nat Immunol 2003;4:471.

8. Lopez CB, Garcia-Sastre A, Williams BR, and Moran TM: Type I interferon induction pathway, but not released interferon, participates in the maturation of dendritic cells induced by negative-strand RNA viruses. J Infect Dis 2003;187:1126.

9. Honda K, Sakaguchi S, Nakajima C, et al.: Selective contribution of IFN-alpha/beta signaling to the maturation of dendritic cells induced by double-stranded RNA or viral infection. Proc Natl Acad Sci U S A 2003;100:10872.

10. Yamamoto M, Sato S, Hemmi H, et al.: Role of adaptor TRIF in the MyD88-independent toll-like receptor signaling pathway. Science 2003;301:640.
11. Sladkova T, and Kostolansky F: The role of cytokines in the immune response to influenza A virus infection. Acta Virol 2006;50:151.

12. Byrnes AA, Ma X, Cuomo P, et al.: Type I interferons and IL-12: convergence and cross-regulation among mediators of cellular immunity. Eur J Immunol 2001;31:2026.

13. McRae BL, Semnani RT, Hayes MP, and van Seventer GA: Type I IFNs inhibit human dendritic cell IL-12 production and Th1 cell development. J Immunol 1998;160:4298.

14. Hoebe K, Janssen EM, Kim SO, Alexopoulou L, Flavell RA, Han J, and Beutler B: Upregulation of costimulatory molecules induced by lipopolysaccharide and doublestranded RNA occurs by Trif-dependent and Trif-independent pathways. Nat Immunol 2003;4:1223.

15. Yoneyama H, Matsuno K, Toda E, et al.: Plasmacytoid DCs help lymph node DCs to induce anti-HSV CTLs. J Exp Med 2005;202:425.

16. Goriely S, Molle C, Nguyen M, et al.: Interferon regulatory factor 3 is involved in Toll like receptor (TLR) 4- and TLR3-induced IL-12p35 gene activation. Blood 2006;107: 1078 .

17. Karp CL, Biron CA, and Irani DN: Interferon beta in multiple sclerosis: Is IL-12 suppression the key? Immunol Today 2000;21:24.

18. van Seventer JM, Nagai T, and van Seventer GA: Interferon-beta differentially regulates expression of the IL-12 family members p35, p40, p19 and EBI3 in activated human dendritic cells. J Neuroimmunol 2002;133:60.

19. Dalod M, Salazar-Mather TP, Malmgaard L, et al.: Interferon alpha/beta and interleukin 12 responses to viral infections: pathways regulating dendritic cell cytokine expression in vivo. J Exp Med 2002;195:517.

20. Cousens LP, Orange JS, Su HC, and Biron CA: Interferonalpha/beta inhibition of interleukin 12 and interferongamma production in vitro and endogenously during viral infection. Proc Natl Acad Sci U S A 1997;94:634.

21. Dalod M, Hamilton T, Salomon R, Salazar-Mather TP, Henry SC, Hamilton JD, and Biron CA: Dendritic cell responses to early murine cytomegalovirus infection: subset functional specialization and differential regulation by interferon alpha/beta. J Exp Med 2003;197:885.

22. Blanco-Quiros A, Gonzalez H, Arranz E, and Lapena S: Decreased interleukin-12 levels in umbilical cord blood in children who developed acute bronchiolitis. Pediatr Pulmonol 1999;28:175.

23. Bont L, Kavelaars A, Heijnen CJ, van Vught AJ, and Kimpen JL: Monocyte interleukin-12 production is inversely related to duration of respiratory failure in respiratory syncytial virus bronchiolitis. J Infect Dis 2000;181:1772.

24. Sinigaglia F, D’Ambrosio D, and Rogge L: Type I interferons and the Th1/Th2 paradigm. Dev Comp Immunol 1999;23:657. 
25. Wang SZ, Bao YX, Rosenberger CL, Tesfaigzi Y, Stark JM, and Harrod KS: IL-12p40 and IL-18 modulate inflammatory and immune responses to respiratory syncytial virus infection. J Immunol 2004;173:4040.

26. Lukacs NW, Tekkanat KK, Berlin A, et al.: Respiratory syncytial virus predisposes mice to augmented allergic airway responses via IL-13-mediated mechanisms. J Immunol 2001;167:1060.

27. Tekkanat KK, Maassab H, Berlin AA, Lincoln PM, Evanoff HL, Kaplan MH, and Lukacs NW: Role of interleukin-12 and stat-4 in the regulation of airway inflammation and hyperreactivity in respiratory syncytial virus infection. Am J Pathol 2001;159:631.
28. Durbin JE, Johnson TR, Durbin RK, Mertz SE, Morotti RA, Peebles RS, and Graham BS: The role of IFN in respiratory syncytial virus pathogenesis. J Immunol 2002;168:2944.

Address reprint requests to: Dr. Nicholas W. Lukacs

Department of Pathology University of Michigan Medical School 1301 Catherine, 5214 Medical Science 1 Ann Arbor, MI 48109

E-mail:nlukacs@umich.edu

Received June 4, 2007; accepted September 4, 2007 


\section{This article has been cited by:}

1. Shelly J. Robertson, Dana N. Mitzel, R. Travis Taylor, Sonja M. Best, Marshall E. Bloom. 2009. Tick-borne flaviviruses: dissecting host immune responses and virus countermeasures. Immunologic Research 43:1-3, 172-186. [CrossRef]

2. Maria Florencia Delgado, Silvina Coviello, A Clara Monsalvo, Guillermina A Melendi, Johanna Zea Hernandez, Juan P Batalle, Leandro Diaz, Alfonsina Trento, Herng-Yu Chang, Wayne Mitzner, Jeffrey Ravetch, José A Melero, Pablo M Irusta, Fernando P Polack. 2009. Lack of antibody affinity maturation due to poor Toll-like receptor stimulation leads to enhanced respiratory syncytial virus disease. Nature Medicine 15:1, 34-41. [CrossRef]

3. Dennis M. Lindell, Thomas E. Lane, Nicholas W. Lukacs. 2008. CXCL10/CXCR3-mediated responses promote immunity to respiratory syncytial virus infection by augmenting dendritic cell and CD8 + T cell efficacy. European Journal of Immunology 38:8, 2168-2179. [CrossRef]

4. M. Shingai, M. Azuma, T. Ebihara, M. Sasai, K. Funami, M. Ayata, H. Ogura, H. Tsutsumi, M. Matsumoto, T. Seya. 2008. Soluble G protein of respiratory syncytial virus inhibits Toll-like receptor 3/4-mediated IFN-beta induction. International Immunology 20:9, 1169-1180. [CrossRef] 